\title{
Hybrid algorithm for acceleration of convergence to cyclic steady state
}

\begin{abstract}
Cyclic process is inherently dynamic, thus it has no steady state. However, the process, after a sufficient number of cycles, will reach a state called Cyclic Steady State (CSS) where the process state variables at some instant within a cycle have the same value at the corresponding instant within each subsequent cycle. Depending on the nature of the cyclic process, the number of cycles needed before the process reaches CSS may vary from tens to thousands. In this study, a hybrid algorithm that aims to predict and accelerate the convergence of the CSS is developed and tested on a cyclic operation of controlled-cycled stirred tank reactor.
\end{abstract}

Keyword: Hybrid algorithm; Prediction of CSS; Acceleration of CSS 